\title{
Prognostic Value of Uric Acid for Pulmonary Thromboembolism
}

\author{
Savas Ozsu MD, Arif M Çoşar MD, Hayriye Bektas Aksoy, Yilmaz Bülbül MD, \\ Funda Oztuna MD, S Caner Karahan, and Tevfik Ozlu MD
}

\begin{abstract}
BACKGROUND: Pulmonary thromboembolism is a serious cardiovascular condition with considerable morbidity and mortality. Clinical studies have indicated that hyperuricemia is an independent risk factor for cardiovascular events. The aim of this study was to investigate possible value of the serum levels of uric acid (UA) in predicting 30-d pulmonary thromboembolism-related mortality. METHODS: Pulmonary thromboembolism was confirmed by computed tomography pulmonary angiography, demographic data, troponin, systolic pressure and pulse on admission, and simplified pulmonary embolism severity index assessment. UA levels were analyzed on admission. The primary end point was all-cause mortality during the first $30 \mathrm{~d}$. RESULTS: A total of 337 acute pulmonary thromboembolism subjects, of whom $59 \%$ were females, were enrolled. The median (interquartile range) serum UA level was $5.35(4.1-7.3) \mathrm{mg} / \mathrm{dL}$. Serum UA levels of deceased subjects were higher than those of alive subjects during the study period $(6.9[4.6-10.0] \mathrm{mg} / \mathrm{dL}$ vs $5.2[4.1-7.0] \mathrm{mg} / \mathrm{dL}, P=.038)$. In the receiver operating characteristic analysis, the area under the curve was 0.650 (CI 0.732-0.960) for UA levels for all-cause mortality. A level of serum UA $\geq 5 \mathrm{mg} / \mathrm{dL}$ showed $73 \%$ sensitivity and $88 \%$ negative predictive value for all-cause $30-\mathrm{d}$ mortality. A weak correlation was determined between the UA levels and age $(r=0.25, P<.001)$ and any troponin $(r=0.267, P<.001)$. Serum UA level was an independent predictor of short-term mortality in pulmonary thromboembolism (odds ratio $1.2, P=.002$ ). CONCLUSIONS: Serum UA levels may be a potential biomarker for predicting outcome in patients with acute pulmonary thromboembolism. Key words: uric acid; pulmonary embolism; prognosis. [Respir Care 2017;62(8):1091-1096. (C) 2017 Daedalus Enterprises]
\end{abstract}

\section{Introduction}

Pulmonary thromboembolism is a serious cardiovascular disease, causing a considerable level of morbidity and mortality. Hemodynamic status, concomitant comorbidities, and dysfunction of right ventricle are predictors of short-term mortality.

Drs Ozsu, Bülbül, Oztuna, and Ozlu are affiliated with the Department of Pulmonary Medicine; Dr Çoşar is affiliated with the Department of Gastroenterology; and Mr Karahan is affiliated with the Department of Clinical Biochemistry, Karadeniz Technical University, School of Medicine, Trabzon, Turkey. Mr Aksoy is affiliated with the Samsun Education and Training Hospital, Samsun, Turkey.

The authors have disclosed no conflicts of interest.

Correspondence: Savas Ozsu MD, Department of Pulmonary Medicine, Karadeniz Technical University School of Medicine, Trabzon 61080, Turkey. E-mail: savasozsu@gmail.com.

DOI: $10.4187 /$ respcare. 05433
Oxidative stress plays an important role in the pathophysiology of many diseases. Levels of uric acid (UA), the final oxidation product of purine metabolism, may rise in conditions of impaired oxidative metabolism, such as diabetes mellitus, idiopathic pulmonary arterial hypertension, chronic thromboembolic pulmonary hypertension, and metabolic syndrome. ${ }^{1-4}$ Clinical studies have also shown that hyperuricemia is an independent risk factor for cardiovascular events. ${ }^{5}$ High serum UA levels have long been known to indicate poor prognosis in some acute diseases. ${ }^{6}$ Hence, the aim of this study was to investigate potential predictive value of serum UA in predicting 30 -d pulmonary thromboembolism-related mortality.

\section{Methods}

\section{Study Design}

Subjects objectively diagnosed with acute pulmonary thromboembolism, according to current guidelines, and 
those age $>18 \mathrm{y}$ were included in this retrospective study, between January 2006 and April 2014. The study was conducted at a university hospital, a tertiary care hospital that serves as a primary referral center for patients with suspected pulmonary thromboembolism. Subjects' data were obtained from our electronic medical record. The study was approved by the local ethics committee.

\section{Subjects and Setting}

The study inclusion criteria included symptomatic pulmonary thromboembolism confirmed by computed tomography pulmonary angiography, demographic data, troponin, systolic pressure and pulse on admission, and simplified pulmonary embolism severity index assessment. The simplified pulmonary embolism severity index was calculated giving one point for the presence of each of the following parameters: (1) age $>80 \mathrm{y}$; (2) presence of a cancer; (3) history of chronic cardiac or pulmonary disease; (4) heart rate $>110$ beats/min; (5) systolic blood pressure 90-100 mm Hg; and (6) arterial oxyhemoglobin saturation $<90 \%$ measured at time of diagnosis of pulmonary embolism. Missing data were considered to be normal. Subjects were divided into 2 groups: low risk $(0$ points) and high risk ( $\geq 1$ point).

Thirty-day all-cause mortality was accepted as the primary end point based on in-hospital deaths or civil registries. The risk stratification was performed according to acute pulmonary embolism guidelines of the European Society of Cardiology 2014. ${ }^{7}$ Due to the deficiency of other markers of right-ventricular dysfunction (eg, echocardiogram and brain natriuretic peptide), subjects with intermediate risk were not classified as with intermediate high or low risk. Systemic hypotension was defined as systemic blood pressure $<90 \mathrm{~mm} \mathrm{Hg}$ or a reduction of at least $40 \mathrm{~mm} \mathrm{Hg}$ for at least $15 \mathrm{~min}$ of follow-up blood pressure. ${ }^{7}$ Patients with renal insufficiency were excluded from the study.

Treatment decisions were made by the physicians caring for the subject, according to the current ESC guidelines and not influenced by the study protocol. Discharged subjects were followed up in the out-patient clinic or were interviewed over the telephone for their latest health conditions.

\section{Biochemical Analysis}

Venous blood samples were analyzed on admission. Troponin- $\mathrm{T}$ or hsTroponin- $\mathrm{T}$ levels were determined by a quantitative electrochemiluminescence assay (cut-off value $<0.010 \mathrm{ng} / \mathrm{mL}$ and cut-off value $\leq 0.014 \mathrm{ng} / \mathrm{mL}$, respectively). A positive troponin test result was defined as a troponin level above the manufacturers' assay threshold for the diagnosis of myocardial injury.

\section{QUICK LOOK}

\section{Current knowledge}

Oxidative stress plays an important role in the pathophysiology of many diseases. Levels of uric acid (UA) is the final oxidation product of purine metabolism. Clinical studies have shown that hyperuricemia is an independent risk factor for cardiovascular events.

\section{What this paper contributes to our knowledge}

It was found that high serum UA level in subjects with pulmonary thromboembolism was an independent predictor of 30-d mortality. Moreover, the increase of the severity of pulmonary thromboembolism was correlated with the increase of the serum UA level. Serum UA level is a simple, easily accessible, and a part of routine laboratory tests. It can be used as a triage marker in the prediction of the risk of pulmonary thromboembolism.

The serum UA levels were measured by the Beckman Coulter A45800 using an enzymatic spectrophotometric method, during the first diagnosis of pulmonary thromboembolism. The normal range of serum UA levels was between 2.6 and $7.2 \mathrm{mg} / \mathrm{dL}$ for male and female sex.

\section{Statistical Analysis}

The Kolmogorov-Smirnov test was used to test for a normal distribution of continuous variables. Data characterized by a normal distribution were expressed as mean and SD. Parameters without such a distribution were expressed as a median with interquartile range. Student $t$ test or Mann-Whitney U test was utilized for comparing the 2 groups. The Kruskal-Wallis (non-normal distribution) test was used for comparing $>2$ groups. Discrete variables were compared using the Fisher exact test (chi-square test). The Spearman test was used to assess the correlation between variables. Sensitivity, specificity, positive predictive value, negative predictive value, and accuracy were analyzed according to standard formulae. The investigation for a prognostic 30-d mortality cutoff value is based on receiver operating characteristic curves. Multivariable logistic regression analysis was used as a stepwise descending method from prognostic factors with a $P$ value significance of $<.1$ in the univariate analysis. $P$ values $<.05$ were considered statistically significant.

\section{Results}

A total of 409 patients were screened for the study, of whom 72 were excluded due to lack of data. The study was 
Table 1. Clinical History and Baseline Characteristics of the Study Subjects

\begin{tabular}{|c|c|c|c|c|}
\hline Characteristics & Total & $\mathrm{UA}<5 \mathrm{mg} / \mathrm{dL}$ & $\mathrm{UA} \geq 5 \mathrm{mg} / \mathrm{dL}$ & $P$ \\
\hline \multicolumn{5}{|l|}{ Demographic factors } \\
\hline Age, median (IQR) y & $70(58-78)$ & $68(53-77)$ & $72(63-79)$ & .005 \\
\hline Male sex, $n(\%)$ & $138(40.8)$ & $53(37.6)$ & $85(43.1)$ & .30 \\
\hline \multicolumn{5}{|l|}{ Clinical parameters, median (IQR) } \\
\hline SBP, median (IQR) mm Hg & $110(100-130)$ & $120(100-130)$ & $110(100-135)$ & .90 \\
\hline DBP, median (IQR) mm Hg & $70(60-80)$ & $70(60-80)$ & $70(60-80)$ & .45 \\
\hline Pulse, median (IQR) beats/min & $92(84-108)$ & $94(84-108)$ & $92(84-110)$ & .97 \\
\hline Breathing frequency, median (IQR) breaths/min & $24(20-26)$ & $22(20-26)$ & $24(20-26)$ & .02 \\
\hline Fever, mean $\pm \mathrm{SD}{ }^{\circ} \mathrm{C}$ & $36.6 \pm 0.6$ & $36.7 \pm 0.5$ & $36.6 \pm 0.7$ & .67 \\
\hline \multicolumn{5}{|l|}{ Comorbidities, $n(\%)$} \\
\hline Malignancy & $48(14.2)$ & $25(17.7)$ & $23(11.7)$ & .11 \\
\hline Cardiopulmonary disease & $55(19.2)$ & $12(9.9)$ & $43(25.9)$ & .001 \\
\hline Deep vein thrombosis $(n=217)$ & $98(45.2)$ & $41(42.7)$ & $57(55.8)$ & .51 \\
\hline \multicolumn{5}{|l|}{ Laboratory biomarkers } \\
\hline D-dimer, median (IQR) $\mu \mathrm{g} / \mathrm{mL}$ & $6(2.7-13.4)$ & $4.6(2.1-9.1)$ & $7.6(3.0-16.4)$ & .001 \\
\hline Arterial oxyhemoglobin saturation, median (IQR) \% $(n=290)$ & $95(91-96)$ & $95(92-97)$ & $94(91-96)$ & .02 \\
\hline Leukocyte, median (IQR) $10^{3} / \mu \mathrm{L}$ & $9.6(7.6-13.0)$ & $8.9(7.6-12.0)$ & $10.2(7.7-13.5)$ & .03 \\
\hline Hemoglobin, mean $\pm \mathrm{SD} g / \mathrm{dL}$ & $12.6 \pm 1.8$ & $12.4 \pm 1.6$ & $12.8 \pm 1.9$ & .052 \\
\hline C-reactive protein, median (IQR) $\mathrm{mg} / \mathrm{dL}(n=312)$ & $5.9(2.1-12.4)$ & $6.5(1.8-14.7)$ & $5.8(2.2-11.0)$ & .36 \\
\hline Elevated troponin, $n(\%)$ & $149(50.7)$ & $44(37.3)$ & $115(59.7)$ & $<.001$ \\
\hline \multicolumn{5}{|l|}{ ESC 2014 algorithm, $n(\%)$} \\
\hline High-risk & $23(6.8)$ & $6(4.3)$ & $17(8.6)$ & .09 \\
\hline Intermediate-risk & $253(74.9)$ & $95(37.5)$ & $158(62.5)$ & .007 \\
\hline Low-risk & $62(18.3)$ & $40(28.4)$ & $22(11.2)$ & $<.001$ \\
\hline sPESI, high-risk & $197(70.1)$ & $85(70.8)$ & $112(69.6)$ & .81 \\
\hline 30-d mortality & $64(19)$ & $18(12.8)$ & $46(23.4)$ & .02 \\
\hline \multicolumn{5}{|l|}{$\begin{array}{l}\mathrm{UA}=\text { uric acid } \\
\mathrm{IQR}=\text { interquartile range } \\
\text { SBP }=\text { systolic blood pressure } \\
\text { DBP }=\text { diastolic blood pressure } \\
\text { sPESI = simplified pulmonary embolism severity index }\end{array}$} \\
\hline
\end{tabular}

completed with 337 subjects. The medical history and baseline characteristics of the subjects are summarized in Table 1.

Pulmonary thromboembolism was diagnosed by computed tomography pulmonary angiography for all subjects. Unfractionated heparin treatment was used in 156 subjects $(46.3 \%)$, whereas low-molecular-weight heparin was used in the other 149 subjects $(44.2 \%)$. Tissue plasminogen activator therapy $(50 \mathrm{mg} / 2 \mathrm{~h})$ was used in 27 subjects $(8 \%)$. In addition, a vena cava filter was inserted into 5 subjects $(1.5 \%)$. Risk factors of pulmonary thromboembolism were immobility (24\%), cancer (14\%), recent history of surgery (12\%), idiopathy (16\%), and various rare causes (34\%).

Serum median (interquartile range) UA level was 5.35 (4.1-7.3) $\mathrm{mg} / \mathrm{dL}$. Subjects with positive troponin had higher UA levels than those with negative troponin (4.85 [3.8$6.45]$ versus 6.35 [4.8-8.2], $P<.001)$. The serum level UA of subjects with high-risk simplified pulmonary embolism severity index was significantly different from that of subjects with low-risk simplified pulmonary embolism severity index (5.2 [95\% CI 3.8-6.6] vs 6.5 [95\% CI
4.5-7.9], $P<.001)$. There was a weak correlation between UA levels and age $(\mathrm{r}=0.25, P<.001)$ and troponin levels $(\mathrm{r}=0.27, P<.001)$.

In receiver operating characteristic analysis, the area under the curve was 0.650 (CI 0.568-0.732) of UA levels for 30 -d all-cause mortality. Serum UA $\geq 5 \mathrm{mg} / \mathrm{dL}$ high showed $73 \%$ sensitivity and $88 \%$ negative predictive value for all-cause 30-d mortality (Table 2).

Serum UA levels were 6.9 (4.8-8.1), 5.5 (4.4-7.65), and $4.3 \mathrm{mg} / \mathrm{dL}(3.4-5.8)$ in high- $(n=23,6.8 \%)$, intermediate- $(n=251,74.5 \%)$, and low-risk $(n=62,18.4 \%)$ pulmonary thromboembolism subjects, respectively. Serum UA levels were statistically significantly higher in subjects with high risk and intermediate risk compared with low-risk pulmonary thromboembolism subjects $(P=.001$ and $P<.001$, respectively) (Fig. 1).

\section{Multivariable Analysis}

Univariate analysis was used to evaluate the possible parameters that affect the 30-d mortality of pulmonary 
Prognostic Value of Uric Acid for Pulmonary Thromboembolism

Table 2. Simplified Pulmonary Embolism Severity Index, Troponin, and Uric Acid Levels for 30-d Mortality

\begin{tabular}{lcccc}
\hline \hline \multicolumn{1}{c}{ Levels } & $\begin{array}{c}\text { Sensitivity } \\
(95 \% \mathrm{CI})\end{array}$ & $\begin{array}{c}\text { Specificity } \\
(95 \% \mathrm{CI})\end{array}$ & $\begin{array}{c}\text { Negative-Pressure } \\
\text { Ventilation (95\% CI) }\end{array}$ & $\begin{array}{c}\text { Positive-Pressure } \\
\text { Ventilation (95\% CI) }\end{array}$ \\
\hline Uric acid, \% & $73(60-83)$ & $45(39-50)$ & $88(81-93)$ & $23(18-30)$ \\
Any positive troponin, \% & $78(65-87)$ & $57(50-63)$ & $90(84-94)$ & $33(26-41)$ \\
SPESI, \% & $92(83-96)$ & $28(22-35)$ & $89(78-95)$ & $35(28-42)$
\end{tabular}

Results are median (interquartile range).

sPESI $=$ simplified pulmonary embolism severity index

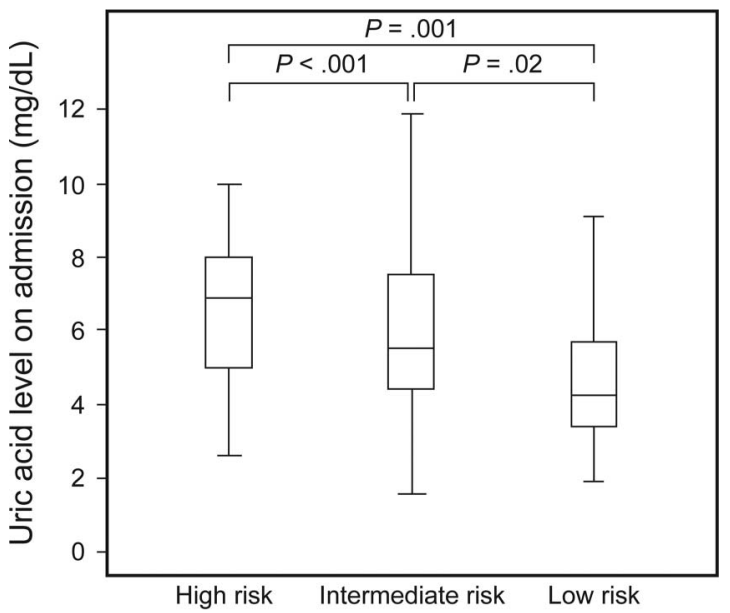

Fig. 1. Serum uric acid (UA) levels of subjects with high-, intermediate-, and low-risk pulmonary thromboembolism. Median (interquartile range) serum UA levels were 6.9 (4.8-8.1), 5.5 (4.4-7.65), and $4.3(3.4-5.8) \mathrm{mg} / \mathrm{dL}$ in subjects with high-, intermediate-, and low-risk pulmonary thromboembolism, respectively. Boxes represent the 25th and 75th percentile, and lines denote the median UA levels. Whiskers represent the minimum and the maximum values of the risk groups.

thromboembolism. Leukocyte count was significant in univariable analysis but was not in multivariable analysis. Multivariable analysis for age, cardiopulmonary disease, cancer history, systolic blood pressure, pulse, and oxygen saturation were not performed because they included the simplified pulmonary embolism severity index model.

Serum UA level was found to be an independent risk factor for 30-d mortality of pulmonary thromboembolism (hazard ratio $1.2, P=.002$ ) (Table 3 ). In addition, when serum UA levels were adjusted with age and cardiopulmonary disease, the adjusted hazard ratio of 30-d mortality for pulmonary thromboembolism was found to be 1.2 (95\% CI 1.1-1.3, $P<.001)$ and $1.2(95 \% \mathrm{CI} 1.1-1.4$, $P<.001)$, respectively.

\section{Clinical Course at 30 Days}

Death at $30 \mathrm{~d}$ occurred in 64 subjects $(19.0 \%, 95 \%$ CI $5.5-8.8 \%)$. Serum UA levels of deceased subjects were higher than for surviving subjects during the study period (5.2 [95\% CI 4.1-7.0] versus 6.9 [95\% CI 4.6-10.0], $P=.038)$. The death rate was $39.1 \%$ in high-risk subjects (95\% CI 20.5-61.2\%), $19.8 \%$ in intermediate-risk subjects (95\% CI $15.2-25.4 \%$ ), and $8.1 \%$ in intermediatelow-risk subjects (95\% CI 5.2-11.0) $(P=.001)$. The death rate was $26.1 \%$ in simplified pulmonary embolism severity index high-risk subjects (95\% CI 20.6-32.6\%) and $5.9 \%$ in simplified pulmonary embolism severity index low-risk subjects (95\% CI 2.8-11.6\%).

\section{Discussion}

In this study, it was found that high serum UA level in subjects with pulmonary thromboembolism is an independent predictor of 30-d mortality. Moreover, the severity of pulmonary thromboembolism was correlated with the serum UA levels. Also, serum UA levels were higher in subjects with positive troponin levels. Therefore, it may be postulated that the serum UA level may be considered as a prognostic marker for patients with pulmonary thromboembolism.

UA is the final oxidation product of purine metabolism. Serum urate levels may be increased in impaired oxidative metabolic conditions. Studies have indicated that UA may induce vascular damage through 3 mechanisms. ${ }^{8}$ First, active oxygen species are produced in the process of UA metabolism, leading to inflammation in vascular endothelial cells and the proliferation of vascular smooth-muscle cells. Second, UA crystals stimulate the production of inflammatory mediators and multinuclear cells, thereby damaging the vascular endothelium. Finally, UA is taken up into vascular endothelial and smooth-muscle cells and then activates an intracellular cascade that finally damages these cells. ${ }^{9}$ Similarly, endothelial damage plays an important role in the coagulation cascade in patients with pulmonary thromboembolism. The relationship between pulmonary thromboembolism and inflammatory markers, such as red cell distribution width and C-reactive protein, was shown before. It has been reported that subjects with pulmonary thromboembolism and high red cell distribution width levels have a higher mortality rate. ${ }^{10}$ In addition, it was found 
Table 3. Predictors of an Adverse 30-Day Mortality

\begin{tabular}{|c|c|c|c|c|}
\hline \multirow{2}{*}{ Characteristics } & \multicolumn{2}{|c|}{ Univariable } & \multicolumn{2}{|c|}{ Multivariable } \\
\hline & $\mathrm{HR}(95 \% \mathrm{CI})$ & $P$ & HR $(95 \% \mathrm{CI})$ & $P$ \\
\hline Age $>80 y$ & $2.9(1.5-5.5)$ & .001 & & \\
\hline Males & $1.2(0.7-2.0)$ & .61 & & \\
\hline Breathing frequency, breaths/min & $1.1(1.1-1.2)$ & $<.001$ & $1.1(1.0-1.1)$ & .02 \\
\hline $\mathrm{SBP}=90-100 \mathrm{~mm} \mathrm{Hg}$ & $2.2(1.3-4.0)$ & .006 & & \\
\hline Heart rate $>110$ beats/min & $1.2(1.1-1.3)$ & $<.001$ & & \\
\hline Pulse $\mathrm{O}_{2}<90 \%$ & $1.6(0.8-3.2)$ & .16 & & \\
\hline Cancer & $3.5(1.8-6.9)$ & $<.001$ & & \\
\hline Cardiopulmonary disease & $2(1.1-3.6)$ & .02 & & \\
\hline DVT & $1.6(0.8-3.3)$ & .23 & & \\
\hline sPESI, high risk & $5.7(2.5-12.8)$ & $<.001$ & $3.6(1.4-9.5)$ & .01 \\
\hline Positive any troponin & $4.550(2.4-8.7)$ & $<.001$ & $2.3(1.1-4.9)$ & .02 \\
\hline Uric acid, mg/dL & $1.3(1.1-1.4)$ & $<.001$ & $1.2(1.1-1.3)$ & .002 \\
\hline D-dimer, & $1.0(1.0-1.0)$ & .47 & & \\
\hline Leukocyte, $10^{3} / \mu \mathrm{L}$ & $1.0(1.0-1.0)$ & .02 & $1.0(1.0-1.0)$ & .32 \\
\hline \multicolumn{5}{|c|}{$\begin{array}{l}\text { Because age, cardiopulmonary disease, cancer history, systolic blood pressure, pulse, and oxygen saturation are included in the simplified pulmonary embolism severity index model, multivariable } \\
\text { analyses were not performed. } \\
\text { HR }=\text { hazard ratio } \\
\text { SBP }=\text { systolic blood pressure } \\
\text { DVT }=\text { deep venous thrombosis } \\
\text { sPESI = simplified pulmonary embolism severity index }\end{array}$} \\
\hline
\end{tabular}

that serum C-reactive protein levels are higher in subjects with pulmonary thromboembolism and with right-ventricular dysfunction. ${ }^{11}$ Considering the similarity of mechanisms, the role of UA in pulmonary thromboembolism is plausible.

The relationship between serum UA levels and the risk of thromboembolic events in subjects with nonvalvular AF was investigated by Numa et al. ${ }^{9}$ It was reported that the risk of a thromboembolic event was increased by 1.45 times independent of serum creatinine level in subjects with a high serum UA level. In another study, Babaoglu et al ${ }^{12}$ investigated the renal and hepatic markers in 98 subjects with acute pulmonary thromboembolism. Similar to our results, they found the lowest and the highest UA levels in subjects with low- and high-risk pulmonary thromboembolism, respectively. Serum UA levels were statistically significant in subjects with intermediate- or high-risk pulmonary thromboembolism, compared with subjects with low-risk pulmonary thromboembolism. However, the relationship between serum UA level and mortality was not evident in this study. ${ }^{12}$

In an experimental study of a pulmonary thromboembolism model in rats, it was observed that serum UA levels increased after pulmonary thromboembolism and normalized in a week. ${ }^{13}$ Similarly, after the use of anticoagulants in patients with pulmonary thromboembolism, serum UA levels may be reduced, and the treatment response can be monitored with serum UA levels. On the other hand, it was reported that allopurinol treatment reduces the risk of cardiovascular disease and the progression of renal disease on the 2-y follow-up. ${ }^{14}$ Further studies investigating the riskreducing effect of allopurinol treatment on the development of pulmonary thromboembolism may be useful.

Elevated UA levels have long been known to be a poor prognostic sign in acute illness. ${ }^{15}$ Several studies have demonstrated that elevated UA levels in pulmonary hypertension correlate with the severity of disease. ${ }^{2,}{ }^{16}$ Serum UA was independently related to mortality on multivariate Cox proportional hazards analysis. Kaplan-Meier survival curves demonstrated that subjects with high serum UA had a significantly higher mortality rate than those with low serum UA. Serum UA levels decrease by successful with prostacyclin treatment of idiopathic pulmonary arterial hypertension patients with prostacyclin. ${ }^{16}$ The mean UA levels were higher in subjects with chronic thromboembolic pulmonary hypertension. ${ }^{3}$

In our study, serum UA levels were found to be higher in deceased subjects, and the UA level was found to be an independent predictor of 30-d mortality in multivariable analysis. The area under the curve was 0.650 in receiver operating characteristic analysis, and the negative predictive value of UA $>5 \mathrm{mg} / \mathrm{dL}$ was $88 \%$ for $30-\mathrm{d}$ mortality. On the other hand, serum UA levels were found to be higher in the subjects with positive troponin levels than in those with normal troponin levels $(6.35$ [95\% CI 4.8-8.2] vs 4.85 [95\% CI 3.8-6.45], $P<.001$ ).

Serum UA levels are dependent on age and sex and are affected by renal impairment and cardiac disease and diuretic therapy; hence, their interpretation may be difficult in some patients. In this study, serum UA levels were high 
in subjects with cardiopulmonary disease. Subjects with serum UA levels $\geq 5 \mathrm{mg} / \mathrm{dL}$ were significantly older as well; however, age and cardiopulmonary disease are included in the simplified pulmonary embolism severity index model. It was shown that if serum UA levels adjust with simplified pulmonary embolism severity index, serum UA levels are significant for 30-d mortality in our study. In addition, all of the subjects were diagnosed with pulmonary thromboembolism by computed tomography, and none of them had renal failure. Although this was a retrospective study, serum UA is routinely measured as part of routine laboratory tests on admission.

\section{Conclusions}

The results of this study indicate that serum UA levels are a promising novel biomarker for predicting treatment outcome in patients with pulmonary thromboembolism. Considering that measurement of serum UA is simple, easily accessible, and a part of routine laboratory tests, it can be used as a triage marker in the prediction of the risk of pulmonary thromboembolism.

\section{ACKNOWLEDGMENTS}

We thank Dr Gökhan Demir for valuable help in preparation of the manuscript. We also thank Prof Ahmet Ayar for critically reading the revision.

\section{REFERENCES}

1. Lindman BR, Dávila-Román VG, Mann DL, McNulty S, Semigran MJ, Lewis GD, et al. Cardiovascular phenotype in HFpEF patients with or without diabetes: a RELAX trial ancillary study. J Am Coll Cardiol 2014;64(6):541-549.

2. Bendayan D, Shitrit D, Ygla M, Huerta M, Fink G, Kramer MR. Hyperuricemia as a prognostic factor in pulmonary arterial hypertension. Respir Med 2003;97(2):130-133.

3. Korkmaz A, Ozlu T, Ozsu S, Kazaz Z, Bulbul Y. Long-term outcomes in acute pulmonary thromboembolism: the incidence of chronic thromboembolic pulmonary hypertension and associated risk factors. Clin Appl Thromb Hemost 2012;18(3):281-288.

4. Ford ES, Li C, Cook S, Choi HK. Serum concentrations of uric acid and the metabolic syndrome among US children and adolescents. Circulation 2007;115(19):2526-2532.

5. Niskanen LK, Laaksonen DE, Nyyssönen K, Alfthan G, Lakka HM, Lakka TA, Salonen JT. Uric acid level as a risk factor for cardiovascular and all-cause mortality in middle-aged men: a prospective cohort study. Arch Intern Med 2004;164(14):1546-1551.

6. Warwick G, Thomas PS, Yates DH. Biomarkers in pulmonary hypertension. Eur Respir J 2008;32(2):503-512.

7. Konstantinides SV, Torbicki A, Agnelli G, Danchin N, Fitzmaurice D, Galiè N, et al. 2014 ESC guidelines on the diagnosis and management of acute pulmonary embolism. Eur Heart J 2014;35(43): 3033-3069.

8. Feig DI, Kang DH, Johnson RJ. Uric acid and cardiovascular risk. N Engl J Med 2008;359(17):1811-1821.

9. Numa S, Hirai T, Nakagawa K, Ohara K, Fukuda N, Nozawa T, Inoue $\mathrm{H}$. Hyperuricemia and transesophageal echocardiographic thromboembolic risk in patients with atrial fibrillation at clinically low-intermediate risk. Circ J 2014;78(7):1600-1605.

10. Ozsu S, Abul Y, Gunaydin S, Orem A, Ozlu T. Prognostic value of red cell distribution width in patients with pulmonary embolism. Clin Appl Thromb Hemost 2014;20(4):365-370.

11. Abul Y, Karakurt S, Ozben B, Toprak A, Celikel T. C-reactive protein in acute pulmonary embolism. J Investig Med 2011;59(1):814.

12. Babaoglu E, Hasanoglu HC, Senturk A, Karalezli A, Kilic H, Aykun G, Oztuna D. Importance of biomarkers in risk stratification of pulmonary thromboembolism patients. J Investig Med 2014;62(2):328331.

13. Zhang YJ, Xu P, Lu WX, Ji YQ, Liu CP, Zhang WH. Study of serum uric acid changes after experimental pulmonary thromboembolism in rats. Zhongguo Wei Zhong Bing Ji Jiu Yi Xue 2005;17(6):342-345.

14. Goicoechea M, Garcia de Vinuesa S, Verdalles U, Verde E, Macias $\mathrm{N}$, Santos A, et al. Allopurinol and progression of CKD and cardiovascular events: long-term follow-up of a randomized clinical trial. Am J Kidney Dis. 2015;65(4):543-549.

15. Woolliscroft JO, Colfer H, Fox IH. Hyperuricemia in acute illness: a poor prognostic sign. Am J Med 1982;72(1):58-62.

16. Voelkel MA, Wynne KM, Badesch DB, Groves BM, Voelkel NF. Hyperuricemia in severe pulmonary hypertension. Chest 2000;117(1): 19-24. 